\title{
Vortex-Breakdown-Induced Particle Capture in Branching Junctions
}

\author{
Jesse T. Ault, ${ }^{1}$ Andrea Fani, ${ }^{2}$ Kevin K. Chen, ${ }^{3}$ Sangwoo Shin, ${ }^{1}$ François Gallaire, ${ }^{4}$ and Howard A. Stone ${ }^{1, *}$ \\ ${ }^{1}$ Department of Mechanical and Aerospace Engineering, Princeton University, Princeton, New Jersey 08544, USA \\ ${ }^{2}$ Dipartimento di Scienze e Tecnologie Aerospaziali, Politecnico di Milano, 20156 Milano, Italy \\ ${ }^{3}$ Department of Aerospace and Mechanical Engineering, University of Southern California, Los Angeles, California 90089, USA \\ ${ }^{4}$ Laboratory of Fluid Mechanics and Instabilities, École Polytechnique Fédérale de Lausanne. EPFL-STI-IGM-LFMI,
} CH-1015 Lausanne, Switzerland

(Received 15 December 2015; revised manuscript received 9 June 2016; published 15 August 2016)

\begin{abstract}
We show experimentally that a flow-induced, Reynolds number-dependent particle-capture mechanism in branching junctions can be enhanced or eliminated by varying the junction angle. In addition, numerical simulations are used to show that the features responsible for this capture have the signatures of classical vortex breakdown, including an approach flow aligned with the vortex axis and a pocket of subcriticality. We show how these recirculation regions originate and evolve and suggest a physical mechanism for their formation. Furthermore, comparing experiments and numerical simulations, the presence of vortex breakdown is found to be an excellent predictor of particle capture. These results inform the design of systems in which suspended particle accumulation can be eliminated or maximized.
\end{abstract}

DOI: 10.1103/PhysRevLett.117.084501

Flows through branching junctions are common in everyday piping systems, industrial applications, and even physiological flows. Despite the prevalence of branching flows and the breadth of studies of these systems, recent discoveries demonstrate new features that have not been studied. For example, for flow through a $T$ junction in which flow enters the base of the $T$ and splits between the two symmetric outlets, it is natural to believe that suspended particles entering the system will find the junction to be a kinematically unstable stagnation region and be swept downstream through the outlets. However, it has been shown that, in fact, bubbles can be trapped in these regions within flow features that resemble vortex breakdown $[1,2]$, which refers to a phenomenon where internal stagnation points develop, followed by regions of reversed flow with limited axial extent [3].

Despite the fact that this capture mechanism depends strongly on the swirling motion of flow in the junction through the interplay of centrifugal, pressure gradient, and drag forces [1], the effect of varying the junction angle has not been explored. We introduce this geometric change, systematically varying the junction angle $\theta$, which introduces significant changes in the secondary swirl velocities [4,5] (Fig. 1). As the Reynolds number Re is increased (while the flow remains laminar), the flow field undergoes qualitative changes involving the formation of internal stagnation regions with strong swirl. We use numerical simulations to show how these features originate and evolve as $\operatorname{Re}$ and $\theta$ are varied, and we show that the physical mechanism for their development is the same as for classical bubble-type vortex breakdown. Finally, we use experiments to demonstrate that the particle capture in two-phase flows is caused by these vortex breakdown features identified in our single-phase simulations.
Experiments were performed in square channels with side length $\ell_{w}$. Particles with density $\rho_{p}$ were suspended in liquids with density $\rho_{f}$ and viscosity $\mu$, which were pumped at average speed $\bar{u}$ through angled junctions with $30^{\circ} \leq \theta \leq 120^{\circ}$ for $0 \leq \operatorname{Re}=\rho_{f} \bar{u} \ell_{w} / \mu \leq 1200$. Three different dilute suspensions were used: bubbles in water $\left(\rho_{p} / \rho_{f} \approx 10^{-3}\right)$, hollow glass beads in water

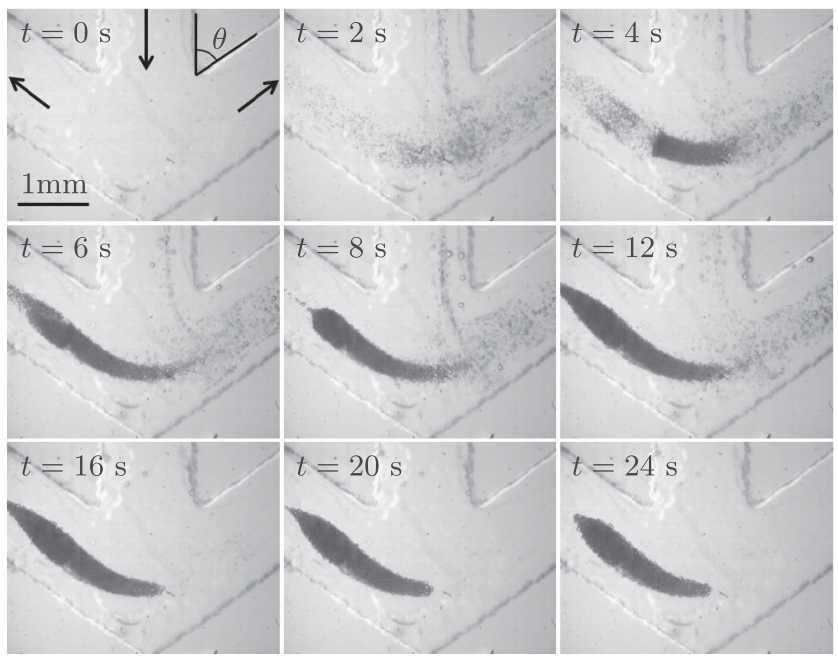

FIG. 1. Bubble capture during flow in branching channels. Water containing $1 \mathrm{~g} / \mathrm{L}$ of sodium sulfate and $0.5 \mathrm{~g} / \mathrm{L}$ of sodium dodecyl sulfate flows through an arrow-shaped junction with $\theta=$ $55^{\circ}$ and $\operatorname{Re}=200$; arrows indicate the flow direction. At $t=1 \mathrm{~s}$, an electric current is switched on to produce bubbles by electrolysis from two wires upstream. The bubbles accumulate in the junction forming a large, stable rotating structure. The bubble source is turned off at $t=14 \mathrm{~s}$, and the bubble cloud takes on a stable shape, remaining in the junction, spinning indefinitely. 

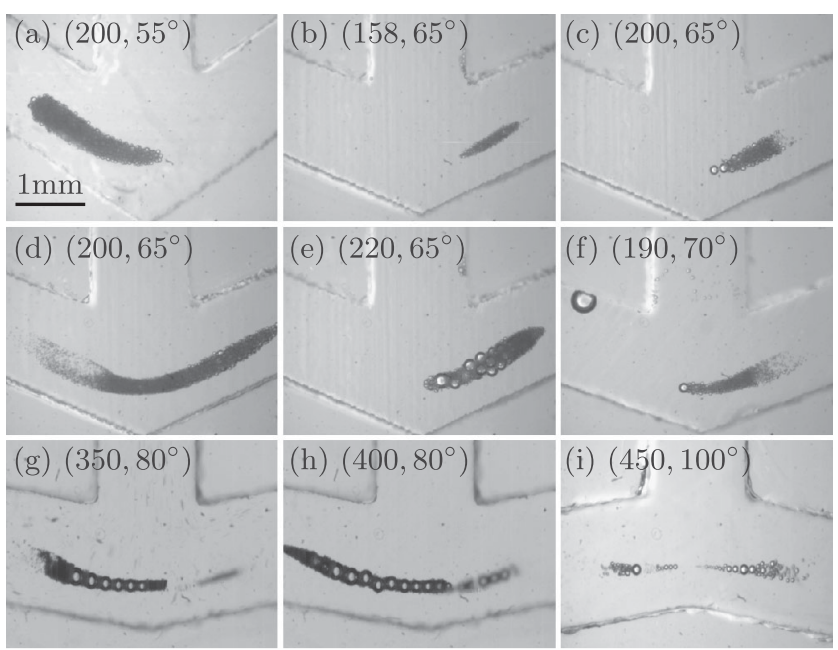

FIG. 2. Junction angle and Reynolds number dependent bubble capture. Values of $\operatorname{Re}$ and $\theta$ are specified in the brackets, respectively. Several capture modes can be identified: (1) A single capture region biased towards one outlet [(a), (b), (c), (e), (f)]. (2) A single capture region extending toward both outlets (d). (3) Two capture regions, either out of plane or in plane, each extending toward one outlet $[(\mathrm{g}),(\mathrm{h})$, (i)]. (4) A final capture mode, not shown, consists of four capture regions, symmetric about both axes of symmetry (see Supplemental Material for videos [6].).

$\left(\rho_{p} / \rho_{f}=0.15\right)$, and polystyrene beads in $0.2 \mathrm{M}$ sodium metatungstate $\left(\rho_{p} / \rho_{f}=0.72\right)$. A typical result for a flow of bubbles $\left(\theta=55^{\circ}, \operatorname{Re}=200\right)$ is shown in Fig. 1 . A stable tubelike structure of bubbles with length $\approx 2 \ell_{w}$ and diameter $\approx 0.35 \ell_{w}$ forms in the neighborhood of the junction, distorts the flow, and may affect the flow downstream or bias the outlet flow rates, although in initial experiments this effect was negligible compared to bias due to small variations in pressure drop between the outlets. At values of Re for which the flow remains steady, these structures remain stable over long time periods. Further details may be found in the Supplemental Material [6].

Experimental results demonstrate a range of possible capture modes (Fig. 2); the most common mode is asymmetric [Figs. 2(a), 2(b), 2(c), 2(e), 2(f)]. Although the dominant feature is a single structure biased toward one outlet, the corresponding single-phase numerical simulations (e.g., Fig. 3) reveal the presence of four vortex breakdown regions, symmetric left to right and front to back. The fact that in the experiments a single, asymmetric particle structure forms, rather than four symmetric structures, likely embodies a supercritical pitchfork bifurcation in the capture process. Generally, smaller values of $\theta$ correspond to asymmetric captures, and larger values correspond to more symmetric capture (Fig. 2). The value of Re does not strongly influence the capture mode.

Numerical simulations were performed using the CFD codes OpenFOAM [7] and Nek5000 [8] to relate the particle

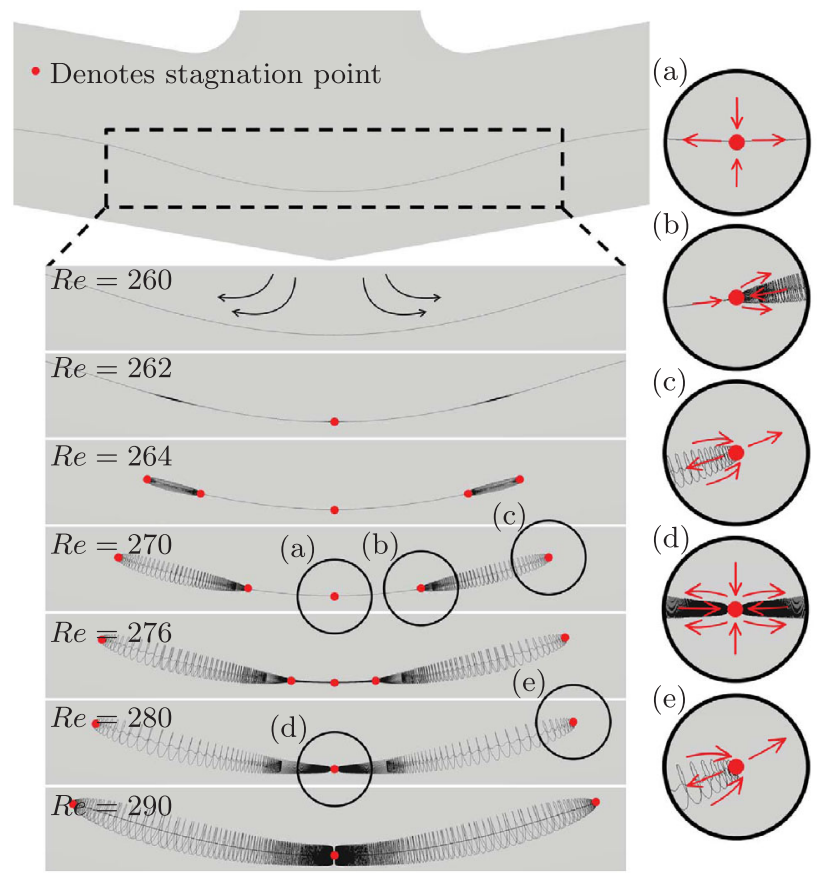

FIG. 3. Steady, single-phase numerical results showing the development of vortex breakdown in an $80^{\circ}$ square-cross-section arrow junction. Bubble-type vortex breakdown regions first appear at $\operatorname{Re}=262$. Each recirculation region is bounded by two stagnation points (SPs), one upstream and the other downstream. These regions grow as Re is increased, merging at the centerplane at $\operatorname{Re} \approx 280$, which represents a second qualitative change in the flow behavior, in which SPs undergo local bifurcations. Red dots mark SPs, arrows indicate the local flow direction, and insets [(a)-(e)] indicate the stability of the SP manifolds. The flow is symmetric about both axes of symmetry, such that four total recirculation regions exist.

capture mechanism to the dynamics of the single-phase flow. For details of the numerical methods see Ref. [2]. Results for $\theta=80^{\circ}$ are presented in Fig. 3, revealing qualitative changes in the flow as $\mathrm{Re}$ is increased. At $\operatorname{Re} \approx 262$, four small, isolated recirculation regions appear, each bounded by upstream and downstream stagnation points. Within these regions the flow recirculates, traveling upstream through the vortex core, strongly resembling the "bubble-form" of vortex breakdown [9]. As Re is increased, the size of the vortex breakdown regions increases (Fig. 3). A shift in the qualitative nature of the recirculation streamlines occurs at $\operatorname{Re} \approx 280$. Below this threshold, the center plane between the two outlets contains stagnation points (SPs) that are stable on the plane and unstable out of plane. The unstable manifolds comprise the axial approach flow toward the four vortex breakdown regions, which are detached from the center plane. As in traditional bubble-type vortex breakdown [10], each breakdown region contains an upstream and downstream SP. The upstream SP is stable on the manifold comprising the vortex axis and unstable on the manifold delimiting the 
boundary of the recirculation region. The downstream SP is the opposite: it is unstable on the vortex axis, sending fluid toward the upstream SP and the outlet, and is stable along the bubble boundary.

Above $\operatorname{Re} \approx 280$ a qualitative shift occurs as the upstream SPs of each vortex merge on the center plane. On each vortex axis, a supercritical pitchfork bifurcation occurs, where two stable SPs at the upstream extents of the breakdown bubbles merge with an unstable SP on the center plane, forming a stable SP on the center plane. Simultaneously, supercritical Hopf bifurcations occur on the center plane. The SPs on the center plane shift from being stable to unstable on that plane, marking the creation of a periodic orbit that is stable on the plane. Out of the plane, the periodic orbit is unstable and marks the boundaries of the recirculation regions. In this case, the approach flow toward the breakdown regions is no longer along the vortex axes, but rather is transverse, having originated from the inlet of the junction.

In flow at a $T$ junction $\left(\theta=90^{\circ}\right)$, Refs. [1] and [2] noted a resemblance with classical bubble-type vortex breakdown, with the primary difference being that the approach flow was perpendicular to the vortex core, whereas in classical bubble-type breakdown the approach flow is aligned with the vortex axis [9]. Here we see that for an angled junction over a range of Re the flow has turned the corner and aligned with the vortex core, e.g., $\operatorname{Re}=264$ in Fig. 3, so for a range of $\operatorname{Re}$ and $\theta$, the flow qualitatively appears the same as the classical bubble-type vortex breakdown.

To confirm the link between the recirculation regions and vortex breakdown, we use numerical simulations to explore the characterization and physical mechanism of the phenomenon. Vortex breakdown [9,11], first observed in the 1950s, remains a subject of some debate and multiple interpretations. It is best understood by considering an axial vortex in uniform unidirectional axial flow. At small values of the swirl number, which compares the maximum tangential velocity to the axial velocity, the vortex diffuses toward the self-similar Batchelor vortex solution composed of a Lamb-Oseen vortex plus a small wake deficit caused by the centrifugal force and low pressure at the vortex center [12]. At larger values of the swirl number, the wake deficit intensifies, yielding a SP preceding a recirculation region (a vortex breakdown bubble). The physical origin of this topological change is debated. Among the existing interpretations, one is based on the reorientation of axial vorticity into azimuthal vorticity through the divergence of vortex tubes [13] while another [14-16] is based on the ability of the inertial waves guided by the vortex core to withstand advection.

Vortices with axial flow are classified according to the propagation of nondispersive axisymmetric neutral Kelvin waves that are supported in the low wave number, inviscid limit. These flows are supercritical if they sustain only downstream traveling waves and subcritical if they sustain both upstream and downstream traveling waves. This criterion is based on the sign of the phase velocity [14], but it can be interpreted as the condition for the existence of a standing wave [15]. When the flow transitions from supercritical to subcritical, downstream disturbances propagate upstream along the vortex and cause vortex breakdown. The mechanism is similar to a hydraulic jump, which also forms when the flow transitions from supercritical to subcritical.

To determine the subcritical or supercritical nature of the vortical structures present in the angled junction flows, simulations with the open source code Nek5000 were used to extract the skeleton of a vortex by monitoring the point of minimum $\lambda_{2}[6,17]$ and in-plane velocity in successive planes along a junction arm; the $\lambda_{2}$ measure is a numerical approach for identifying vortex cores. The subcritical or supercritical nature of the local flow has been assessed similar to Ref. [18], except that we first axisymmetrized the velocity distribution and were limited radially by the proximity of the walls. We chose junctions with $\theta=80^{\circ}$, $90^{\circ}$, and $100^{\circ}$. Figure 4 shows the evolution of the axial vorticity $\omega$, nondimensionalized by $\bar{u} / \ell_{w}$, on the vortex center as a function of the downstream coordinate $x$ along an outlet. The vorticity is not strongly influenced by Re at

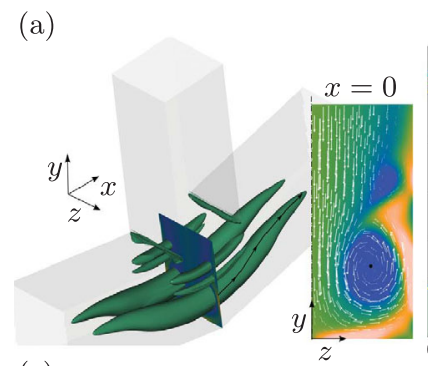

(b)
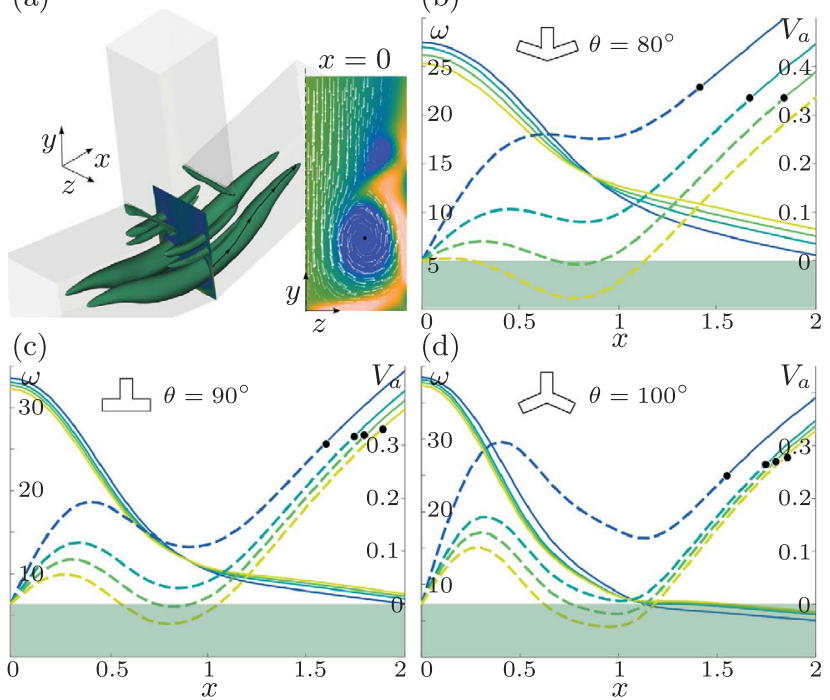

(d)

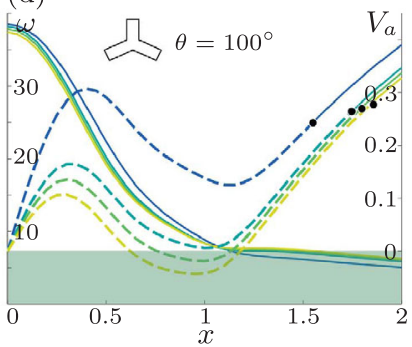

FIG. 4. (a) Vortices depicted by the $\lambda_{2}$ criterion [6,17] for a junction with $\operatorname{Re}=240$ and $\theta=80^{\circ}$. (b)-(d) Axial vorticity $\omega$ (solid lines) and axial velocity $V_{a}$ (dashed lines) as a function of the $x$ position along a junction arm for (b) $\theta=80^{\circ}$, (c) $90^{\circ}$ and (d) $100^{\circ}$. The blue, teal, green, and yellow lines correspond to Re of (b) 210, 240, 260, 270, (c) 280, 300, 310, 320, and (d) $340,380,390,400$, respectively. The axial velocity lines are dashed to denote the extent of the subcritical region, the limits of which are marked by the black dots. The presence of vortex breakdown is indicated by negative regions of $V_{a}$. Vortex breakdown occurs when the extent of the subcritical region is greater than $x \approx 1.8$. 
$x=0$, but does depend on $\theta$. Surprisingly, for angles close to $90^{\circ}$ the vorticity is more intense when $\theta$ is larger. In contrast, Dean vortices increase in strength with increasing channel curvature, showing the complexity and threedimensional nature of the secondary vorticity generation mechanisms in branching junctions. The results also show that $\omega$ is maximal at $x=0$ on the symmetry plane, diminishing downstream because of diffusion.

Next, we show the evolution of the axial velocity $V_{a}$, nondimensionalized by $\bar{u}$, on the axis as a function of $x$ for different Re and $\theta$ [Figs. 4(b)-4(d)]. The non-monotonic behavior follows three stages. Near the symmetry plane $x=0$, where $V_{a}$ is zero, the axial velocity first increases significantly due to entrainment by the flow, which follows the geometry. However, because of the nonlinear effect of the reorientation of vorticity [13], the wake is reinforced and $V_{a}$ decreases further downstream. Finally, the wake deficit is filled in as the vorticity weakens and the axial momentum diffusion becomes dominant.

As a consequence of the combined variations of $\omega$ and $V_{a}$ with $x$, the flow transitions from a high-vorticity lowvelocity configuration into a low-vorticity high-velocity state, which defines a subcritical region of finite extent [Figs. 4(b)-4(d), dashed lines]. The existence of a pocket of subcriticality at the inlet contrasts with most swirling jet studies where the flow is supercritical at the inlet, and transition to subcriticality is triggered by an adverse pressure gradient or by the pipe geometry. The pocket of subcriticality was, however, anticipated by Benjamin [19], who analyzed subcritical flows and concluded that the existence of a sufficiently large region of subcriticality was a necessary condition for vortex breakdown onset. The result is reminiscent of the criterion that a sufficiently large pocket of absolute instability is necessary for a flow to become globally unstable [20]. In both cases, the determination of the pocket of subcriticality or absolute instability is problem dependent and difficult to estimate, in particular when the flow cannot be reasonably approximated as parallel.

The above reasoning and observations suggest that the physical mechanism resulting in the formation of recirculation regions in the flow through branching junctions is the same as the classical forms of vortex breakdown, where swirl and Re can be controlled independently. In both cases, the subcritical nature of the flow is responsible for the recirculation formation. In the present junction flows, the wake deficit is enhanced by the tilting of the axial vorticity, along the lines of the Brown-Lopez mechanism [13], extending the subcritical region sufficiently for vortex breakdown to occur. The analyses in Fig. 4 at $\theta=80^{\circ}$, $90^{\circ}$, and $100^{\circ}$ suggest that vortex breakdown occurs when the subcritical region extends beyond $x \approx 1.8$, which occurs at a Re that depends on $\theta$.

The hydraulic jump analogy is also interesting because it reveals that flows transitioning from subcritical to supercritical are expected to transition smoothly without discontinuity. However, sufficiently extended subcritical flows can sustain standing waves of finite amplitude, suggesting the following scenario: if the flow becomes supercritical after a distance (healing length) which is short in comparison to sustainable standing waves, nothing unusual will happen. However, if the flow remains subcritical over a distance comparable to the wavelength of admissible standing waves, then vortex breakdown can happen, as a consequence of a trapped wave.

Now, comparing experiments and numerical simulations, we show that the presence of vortex breakdown in singlephase flows is an excellent predictor of particle capture in dilute two-phase systems. A phase diagram showing the experimental capture limits is shown in Fig. 5. The lower and upper Re limits for capture were found using particleto-fluid density ratios of $\rho_{p} / \rho_{f}=0.72,0.15$, and $\approx 10^{-3}$. The capture limits are not seen to depend strongly on $\rho_{p} / \rho_{f}$. A maximum particle density of $\rho_{p} \approx 0.7 \rho_{f}$ for capture in a $T$ junction has been suggested [1]. Figure 5 may be used to design systems that maximize or suppress particle capture by selecting appropriate values of Re and $\theta$. Qualitatively, the most particles appear to be trapped around $\mathrm{Re}=230$ and $\theta=70^{\circ}$.

The minimum Re for which vortex breakdown occurred in single-phase numerical simulations is shown in Fig. 5 as the thick, colored line. For $\theta>65^{\circ}$ (solid red line), vortex breakdown develops before the flow becomes unsteady. However, for $\theta<65^{\circ}$ (dashed blue line), vortex breakdown only develops for $\mathrm{Re}$ at which the simulated flow is unsteady. The minimum Re for onset of vortex breakdown

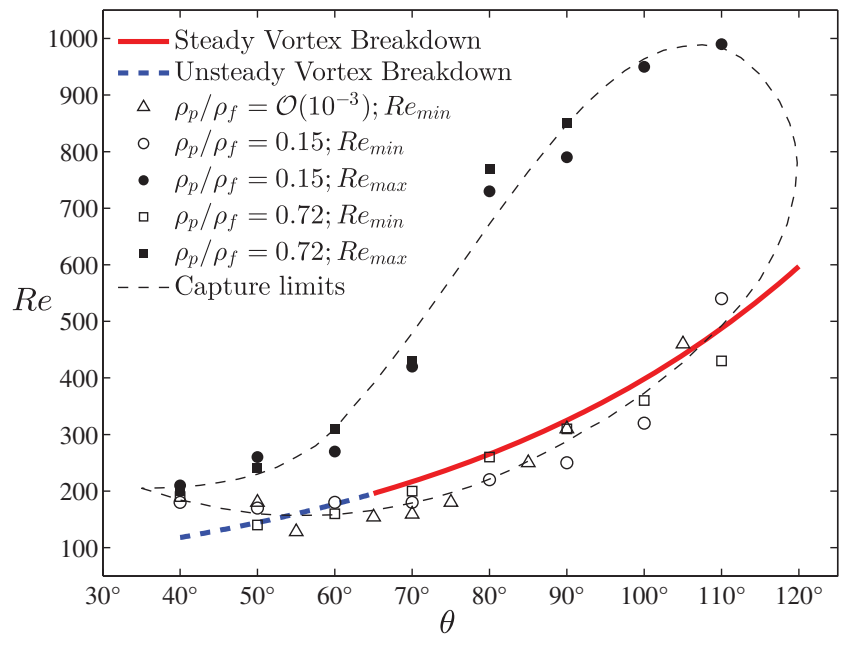

FIG. 5. Phase diagram showing the limits of particle capture. The solid red line denotes the minimum Re at which vortex breakdown occurred in our single-phase numerical simulations. Below $\theta=65^{\circ}$ the flow acquires an unsteady periodic oscillation before the onset of vortex breakdown, and so the vortex breakdown is oscillatory for those junctions. The minimum $\mathrm{Re}$ for the onset of this oscillatory vortex breakdown for $\theta<65^{\circ}$ is projected as the dashed blue line. 
corresponds strongly with the experimental minimum Re for particle capture, suggesting that the onset of vortex breakdown is necessary and responsible for particle capture and that particle-fluid hydrodynamic interactions are not critical to the initial stages of trapping for the particle sizes and densities of interest here. Thus, single-phase numerical simulations can be used to predict the onset of capture in dilute two-phase systems.

The particle capture mechanism studied here for flow in angled junctions has potential impact across a range of applications. For example, this mechanism has recently been incorporated into a new technique to produce captured giant unilamellar vesicles [21]. Furthermore, these results allow systems to be designed in which particle capture can be enhanced or entirely avoided. We have emphasized that the recirculation phenomenon seen in these common branching flows is the same as the classical vortex breakdown phenomenon. Finally, we recognize that similar configurations, such as the cross-slot flow, yield additional symmetry breaking bifurcations as Re is increased [22].

*hastone@princeton.edu

[1] D. Vigolo, S. Radl, and H. A. Stone, Proc. Natl. Acad. Sci. U.S.A. 111, 4770 (2014).

[2] K. K. Chen, C. W. Rowley, and H. A. Stone, Phys. Fluids 27, 034107 (2015).

[3] S. Leibovich, Annu. Rev. Fluid Mech. 10, 221 (1978).

[4] W. R. Dean, Philos. Mag. 4, 208 (1927).
[5] W. R. Dean, Philos. Mag. 5, 673 (1928).

[6] See Supplemental Material at http://link.aps.org/ supplemental/10.1103/PhysRevLett.117.084501 for additional information.

[7] H. G. Weller, G. Tabor, H. Jasak, and C. Fureby, Comput. Phys. 12, 620 (1998).

[8] P. F. Fischer, J. W. Lottes, and S. G. Kerkemeier, nek5000 Web page, 2008), https://nek5000.mcs.anl.gov.

[9] M. G. Hall, Annu. Rev. Fluid Mech. 4, 195 (1972).

[10] T. Sarpkaya, J. Fluid Mech. 45, 545 (1971).

[11] S. Leibovich, AIAA J. 22, 1192 (1984).

[12] G. K. Batchelor, J. Fluid Mech. 20, 645 (1964).

[13] G. Brown and J. Lopez, J. Fluid Mech. 221, 553 (1990).

[14] H. Squire, Analysis of the Vortex Breakdown Phenomenon (Imperial College of Science and Technology, Aeronautics Department, London, 1960).

[15] T. B. Benjamin, J. Fluid Mech. 14, 593 (1962).

[16] S. Wang and Z. Rusak, J. Fluid Mech. 340, 177 (1997).

[17] J. Jeong and F. Hussain, J. Fluid Mech. 285, 69 (1995).

[18] D. Darmofal and E. Murman, On the Trapped Wave Nature of Axisymmetric Vortex Breakdown, 94-2318 (AIAA, 1994), pp. 1-28.

[19] T. B. Benjamin, J. Fluid Mech. 28, 65 (1967).

[20] J.-M. Chomaz, P. Huerre, and L. G. Redekopp, Stud. Appl. Math. 84, 119 (1991).

[21] S. Shin, J. T. Ault, and H. A. Stone, Langmuir 31, 7178 (2015).

[22] S. J. Haward, R. J. Poole, M. A. Alves, P. J. Oliveira, N. Goldenfeld, and A. Q. Shen, Phys. Rev. E 93, 031101-1 (2016). 